\title{
Analysis of cosmic ray dynamics and ionospheric parameters during increased solar activity and magnetic storms
}

\author{
Oksana Mandrikova*, Yuryi Polozov, and Bogdana Mandrikova \\ Institute of Cosmophysical Research and Radio Wave Propagation FEB RAS, 684034, Kamchatskiy \\ krai, Paratunka, Russian Federation
}

\begin{abstract}
Based on the ground station data, the paper investigates the dynamics of space rays and ionospheric parameters during strong and moderate magnetic storms 2018. The research was carried out applying new methods for modeling and data analysis suggested by the authors. In the course of investigation, anomalous changes in ionospheric parameters preceding and accompanying magnetic storms were detected. When they were compared with the data of cosmic rays and interplanetary magnetic field, correlation was observed. It confirms the solar nature of the detected ionospheric effects. Application of regression methods confirmed the assumption on statistically significant effect of interplanetary magnetic field parameters on ionospheric parameter dynamics during increased solar activity. The results are of interest in the tasks of space weather forecast.
\end{abstract}

\section{Introduction}

Investigation of ionospheric parameters and space weather data is of interest in the tasks of solar-terrestrial physics and applied studies associated with space weather. Changes of the conditions on the Sun, in the solar wind, Earth magnetosphere and ionosphere significantly affects the operation and reliability of satellite and ground technological systems and threatens human health and lives. By the present time, the problem of real-time and accurate forecast of space weather has not been solved [1]. It is very important to create automatic methods for recorded data analysis to make online forecast of space weather.

In order to investigate space ray dynamics, space and ground data of neutron monitor global network data are used [2]. The recorded variations of cosmic rays are a complicated nonlinear dependence. On the Earth surface, cosmic ray intensity depends on air temperature, pressure, observation site latitude and geomagnetic field state, electromagnetic situation in the solar system and physical conditions in the Galaxy [1]. Initial cosmic ray variation amplitude depends on particle energy and interplanetary magnetic field strength. Classical methods for neutron monitor data analysis (spectral methods [3], smoothed methods [4]) are not effective enough. Modern methods, for example station circle method [5] allows one to determine the basic characteristics of cosmic ray variations with acceptable accuracy. However, these methods require labor-consuming calculations and they have not been automatized. Taking into account incomplete knowledge on the processes in the near-earth space and, as a consequence, on the recorded data inner

*Corresponding author: oksanam1@mail.ru 
structure, the paper applies neural network apparatus to analyze neutron monitor data. It is known that neural networks are capable of approximating data complicated non-linear dependences without complete a priori knowledge on inner relations and dependences [6]. A significant advantage of this apparatus is the possibility of numerical realization that is important for the tasks of real-time data analysis. The approach used in the paper was firstly suggested in the paper [7] and is based on the application of neural networks of vector quantization [8]. This paper is the continuation of the investigations in this directions. The results confirmed the possibility of application of the developed approach in the problems of detection of anomalous changes in cosmic ray dynamics including those of low amplitude.

Cosmic ray data were analyzed together with ionospheric parameters in the paper. Ionospheric parameter regular changes depend heavily on the time of a day and season, on solar and geomagnetic activities, geographical location (polar and aurora zones, midlatitudinal and equatorial regions) and on other factors [9]. The most prominent irregular changes are observed in the ionosphere during solar flare events and magnetic disturbances and cause ionospheric inhomogeneity formations (ionospheric storms). Ionospheric storms have complicated spatial-time distribution and may have positive and negative phases [9]. The tasks of ionospheric data analysis and ionospheric process investigations are solved by many authors [9]. Among the main approaches, it is possible to emphasize the meridian method, physical models, ionosphere empirical models and neural network models [9]. Application of traditional methods based on smoothing techniques allows us to study data stable characteristics but causes losses of information on fast nonstationary changes [10]. The efficiency of modern methods (empirical, physical and neural network ones) is determined by the presence of qualitative real-time data of geophysical monitoring and decreases significantly during disturbed periods. The approach used at the laboratory is based on the application of wavelet transform and adaptive threshold functions. The developed calculation solutions has multiply proved their efficiency in the problems of ionospheric data real-time analysis and detection of ionospheric disturbances of different intensity $[10,11]$. The results of the work showed high sensitivity of the developed method and the possibility to apply it in the problems of geophysical data complex analysis and detection of space weather effects. In the course of investigation, we confirmed the possibility of occurrences of weak anomalous changes in ionospheric parameters preceding and accompanying magnetic storm periods. When compared with cosmic ray data and interplanetary magnetic field parameters, correlation was noticed confirming solar nature of detected ionospheric effects. Application of regression methods confirmed the suggestion on statistically significant effect of interplanetary magnetic field parameters on ionosphere dynamics during increased solar activity.

\section{Description of methods and algorithms}

\subsection{Multicomponent model and ionospheric parameter analysis algorithm}

Ionospheric parameter time series can be represented in the form [11]

$$
\mathrm{f}(\mathrm{t})=\mathrm{A}(\mathrm{t})+\mathrm{U}(\mathrm{t})+\mathrm{e}(\mathrm{t})=\sum_{\mu=\overline{1, T}} \alpha^{\mu}(\mathrm{t})+\sum_{\eta} \beta_{\text {dist }}^{\eta}(\mathrm{t})+\mathrm{e}(\mathrm{t})
$$

where $A(t)=\sum_{\mu=\overline{1, T}} \alpha^{\mu}(t) \quad(\mu=\overline{1, T}$ is the component number) is the model regular component describing ionospheric parameter characteristic variations, the components $\alpha^{\mu}(t)$ are determined by local factors and include seasonal variations, diurnal oscillation etc.; $U(t)=\sum_{\eta} \beta_{\text {dist }}^{\eta}(t)$ is the model anomalous component describing nonstationary shortperiod changes in ionospheric parameters during disturbed periods (oscillations during increased solar activity, magnetospheric disturbances etc.), it is assumed that the 
component $U(t)=0, e(t)$ is a noise one during ionospheric calm periods. The technique for identification of model (1) components was described in the papers [11, 12]. In this paper we use an anomalous component of the model $U(t)$, identification of which is based on the application of threshold functions [11]:

$$
\begin{gathered}
\mathrm{U}(\mathrm{t})=\sum_{\eta} \beta_{\text {dist }}^{\eta}(\mathrm{t})=\sum_{\eta, \mathrm{n}} \mathrm{P}_{1, \eta}\left(\mathrm{d}_{\eta, \mathrm{n}}\right) \Psi_{\eta, \mathrm{n}}(\mathrm{t})+\sum_{\eta, \mathrm{n}} \mathrm{P}_{2, \eta}\left(\mathrm{d}_{\eta, \mathrm{n}}\right) \Psi_{\eta, \mathrm{n}}(\mathrm{t}) \\
\mathrm{P}_{1, \eta}(\mathrm{x})=\left\{\begin{array}{r}
0, \text { if }|\mathrm{x}| \leq \mathrm{T}_{1, \eta} \mathrm{or}|\mathrm{x}|>\mathrm{T}_{2, \eta} \\
\mathrm{x}, \text { if } \mathrm{T}_{1, \mathrm{n}}<|\mathrm{x}| \leq \mathrm{T}_{1, \eta}
\end{array}\right. \\
\mathrm{P}_{2, \eta}(\mathrm{x})=\left\{\begin{array}{l}
0, \text { if }|\mathrm{x}| \leq \mathrm{T}_{2, \eta} \\
\mathrm{x}, \text { if }|\mathrm{x}|>\mathrm{T}_{2, \eta}
\end{array}\right.
\end{gathered}
$$

where the coefficients $\mathrm{d}_{\eta, \mathrm{n}}=\left\langle\mathrm{f}, \Psi_{\eta, \mathrm{n}}\right\rangle,\left\{\Psi_{\eta, \mathrm{n}}\right\}_{\eta, \mathrm{n} \in \mathrm{Z}}$ are orthogonal wavelet basis of space $\mathrm{L}^{2}(\mathrm{R})$, threshold $T_{1, \eta}$ determines ionospheric disturbances of scale $\eta$ of low intensity, threshold $T_{2, \eta}$ determines ionospheric disturbances of scale $\eta$ of high intensity.

Taking into account the ionospehric process changeability, we introduced adaptive thresholds $\mathrm{P}_{\mathrm{i}, \eta}^{\mathrm{ad}}, \mathrm{i}=1,2$ and coefficients $\mathrm{d}_{\eta, \mathrm{n}}($ ratio $(2))$ are accepted to be equal

$$
d_{\eta, n}=\left\{\begin{array}{c}
d_{\eta, n}^{i+}, \text { if }\left(d_{\eta, n}-d_{\eta, n}^{\text {med }}\right) \geq P_{i, \eta}^{\text {ad }} \\
d_{\eta, n}^{i-}, \text { if }\left(d_{\eta, n}-d_{\eta, n}^{\text {med }}\right) \leq-P_{i, \eta}^{\text {ad }}
\end{array}\right.
$$

where $P_{i, \eta}^{a d}=V_{i} * S t_{\eta}, S t_{\eta}=\sqrt{\frac{1}{\Phi-1} \sum_{n=1}^{\Phi}\left(d_{\eta, n}-\overline{d_{\eta, n}}\right)^{2}}, \overline{d_{\eta, n}}$ and $d_{\eta, n}^{\text {med }}$ are the average and the median, respectively, which are calculated taking into account the diurnal variation of ionospheric data within the moving time window of the length $\Phi$.

Algorithm for detection and calculation of anomalous changes in ionospheric parameters is based on the analysis of model $\mathrm{U}(\mathrm{t})$ anomalous component errors:

- We think that an anomaly of scale $\eta_{0}$ occured in the ionosphere at a time instant $n=n_{0}$ if $\left|d_{\eta_{0}, n_{0}}\right|>P_{i, \eta_{0}}^{\mathrm{ad}}$.

- Anomaly $I_{\eta_{0}, n_{0}}$ intensity at a time instant $n_{0}$ on scale $\eta_{0}$ is determined as $I_{\eta_{0}, n_{0}}=$ $\left|\mathrm{d}_{\eta_{0}, \mathrm{n}_{0}}\right|$.

- If $\forall n_{s} \in\left(t_{1}, t_{2}\right), t_{2}>t_{1}:\left|d_{\eta_{0}, n_{s}}\right|>P_{i, \eta_{0}}^{a d}$, then we consider that during the time interval $\left(t_{1}, t_{2}\right)$, an anomaly of scale $\eta_{0}$ was observed, its duration is determined as $l_{\eta_{0}}=t_{2}-t_{1}$.

\subsection{Method for neutron monitor data classification based on LVQ neural network}

\subsubsection{Data pre-processing based on multiscale wavelet decomposition:}

- Assuming that the initial time series $f_{j}(t)$ belongs to a space with resolution $j=0$, based on the multiscale wavelet decomposition $f_{0}(t)$ to the level $m$ we obtain [13]:

$$
f_{0}(t)=g_{-1}(t)+g_{-2}(t)+\ldots+g_{-m}(t)+f_{-m}(t)=\sum_{k=-1 n}^{-m} \sum_{k, n} d_{k, n}(t)+\underset{n}{\sum} c_{-m, n} \phi_{-m, n}(t)
$$

components g-i are detailing ones (are not used in the paper); component $f_{-m}(t)=\sum_{n} c_{-m, n} \phi_{-m . n}(t)$ is a smoothed one, $c_{j, n}=\left\langle f, \phi_{j, n}\right\rangle, d_{j, n}=\left\langle f, \Psi_{j, n}\right\rangle$; 
$\Psi_{j, n}(t)=2^{j / 2} \Psi\left(2^{j} t-n\right)$ is the basic wavelet, $\phi_{-m, n}(t)=2^{-m / 2} \phi\left(2^{-m} t-n\right)$ is the scaling function.

- In order to recover the initial resolution of a function we perform wavelet reconstruction operation [13]: $f_{0}^{(-m)}(t)=\sum_{n} c_{0, n}^{(-m)} \phi_{0, n}(t)$, (the superscript $\quad(-m)$ corresponds to the function resolution before the wavelet reconstruction operation).

- To exclude the boundary effect influence, we remove the first and the latest $2^{m} * q$ counts from the reconstructed set (vicinity size, containing the boundary effect at the decomposition level $m$, is determined by the formula $h_{j}=2^{j} * q$, where $q$ is the carrier applied by the basic function).

\subsubsection{Architecture and operating principles for LVQ neural network}

LVO neural network consists of 2 layers. The architecture applied in the work is illustrated in Fig. 1 [6]. The first layer is the Kohonen one (competitive layer [8], Fig. 2), the second layer is a linear one and it is formed during the neural network training. The second layer determines the correspondence between the neuron numbers of the 1-st layer (clusters) $\mathrm{k}$ and respective classes $l[8]$

$$
F_{l}=\sum_{k} w_{k l} y_{k}
$$

where $w_{k l}$ are the weight coefficients of neuron $l$ of the network second layer associated with neuron $k$ of the network first layer, $y_{k}$ is the output value of neuron $k$ of the network first layer.

Input vector clustering into a defined number of classes is carried out with the metrics

$$
d_{k}=d\left(X, W_{k}\right)=\left\|X-W_{k}\right\|=\sqrt{\sum_{i=1}^{I}\left(x_{i}-w_{i k}\right)^{2}}
$$

where $X$ is the input vector; $W_{k}$ is the weight vector of neuron $k$ of the first competitive layer, $I$ is the input vector dimension. 


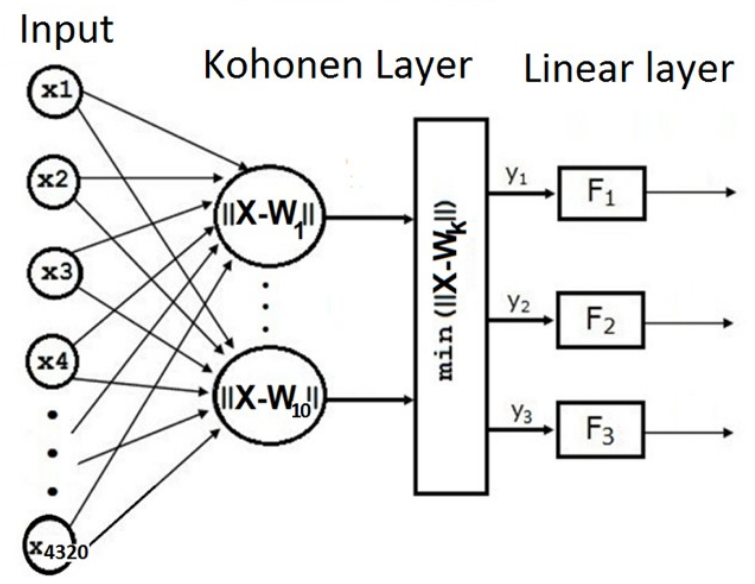

Fig. 1. LVO neural network structure.

In the course of neural network $(\mathrm{NN})$ operation, based on the distance $d_{k}$ estimate (ratio (4)), winning neuron $p$ is determined for which

$$
D=d_{\min }\left(X, W_{k}\right)=\min _{k}\left\|X-W_{k}\right\| \text {. }
$$

Output value of the winning neuron $p$ is set to be equal to 1: $y_{p}=1$, and other neurons are equal to zero: $y_{k}=0, k \neq p$. The winning neuron sets the belonging of an input vector to $\mathrm{X}$ class associated with this neuron (cluster).

NN output vector has the dimension equal to the number of classes $L$ (in the paper $L=3$, classes are described below). Cluster number was chosen empirically and was equal to 20 . In case of correctly a trained network, one element of an output vector is equal to 1 and the rest are zero. Thus, NC allows us to solve the problem of belonging of an output vector to one of a priori known classes.

\subsubsection{Determination of neural network classes and a scheme to solve the problem of data classification}

According to the problem under investigation, we defined the following NN classes in the paper $[7,14]$ :

- «Calm» class - absence of sporadic layers. «Calm» class is characterized by (1) absence of active spots and flares on the Sun (zero flare activity); (2) absence of solar wind flux from the visible side on the line with the Earth; (3) absence of magnetic storms and disturbances in the magnetosphere (geomagnetic activity index (K-index) is $\leq 2$ ).

- «Weakly-disturbed» class - presence of sporadic effects of low amplitude. «Weaklydisturbed» class is characterized by (1) insignificant flares on the Sun directed to the Earth; (2) weak disturbances in the magnetosphere (K-index is 3,4$)$.

- «Disturbed» class - presence of sporadic layers of high amplitude. «Disturbed» class is characterized by: (1) penetration of disturbed high-velocity fluxes of solar wind and/or a shock wave associated with it to the Earth near-space; (2) magnetic storm occurrence and presence of strong disturbances in the magnetosphere ( $\mathrm{K}$-index is $\geq 5$ ).

Based on the suggested approach, solution of data classification problem can be represented in the form of a scheme illustrated in Fig. 2. 


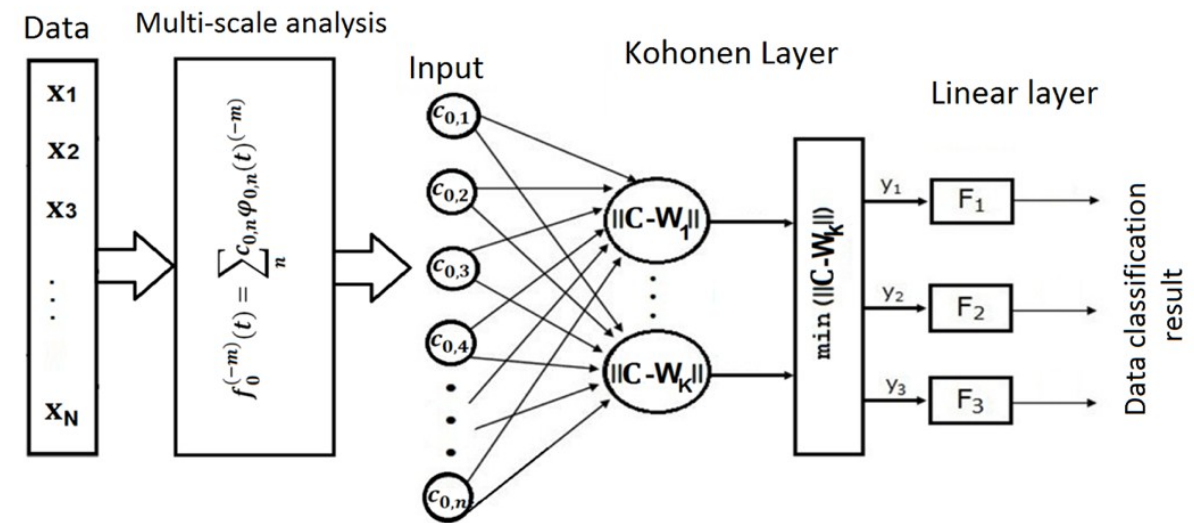

Fig. 2. Scheme of the solution of neutron monitor data classification problem.

\section{Results of method application}

In the experiments we applied neutron monitor minute data from Moscow station [2], analyzed strong and moderate storms occurred in 2018. When constructing a training sampling, data selection for each introduced class (Section 2.2.3) was based on the analysis of geomagnetic activity indicators, A-, K- and Dst-indexes were used [15]. The «calm» class was formed from the data for those time intervals during which A-index was $<7, \mathrm{~K}$ index was $<3$, Dst-index was within \pm 4 . The «weakly-disturbed» class was formed from the data for those time intervals during which A-index was $<18$, K-index was $<5$, Dstindex was within \pm 8 . The «disturbed» class included the time intervals during which Aindex was $>18$, K-index was $>5$, Dst-index was out of \pm 8 . We used 20 vectors to train the neural networks, they are: 10 vectors, the geomagnetic indexes of which corresponded to the «calm» class; 6 vectors corresponded to the «weakly disturbed» class; 4 vectors corresponded to the «disturbed» class. To test the network, we used 36 vectors (data were not used during network training), they are: 10 vectors, the geomagnetic indexes of which corresponded to the «calm» class; 21 vectors corresponded to the «weakly disturbed» class; 5 vectors corresponded to the «disturbed» class. Each vector is a three-day interval containing minute data of neutron monitor (NM). Data multi-scale wavelet decompositions were carried out with Koiflet wavelet functions, the choice of which was explained in the paper [16]. According to the work [16] function expansion was carried out to the expansion 1-st level. The results with the least error were obtained applying the Koiflet wavelet functions of order 3. Taking into account the NM data diurnal variation and with the aim of the possibility of processing the data in a moving time window (we used the time window step equal to 3 hours), 8 neural networks were constructed. On each of them we supplied the input vectors of the size of $\mathrm{N}=4320$ counts (corresponds to three days) with the displacement of $\mathrm{M}=180$ counts (corresponds to 3 hours).

Fig. 3 illustrates the results of data processing during a strong magnetic storm on August 25-27, 2018. Based on space weather data [15] owing to the weakening of coronal hole, solar wind velocity (SWV) decreased from $510 \mathrm{~km} / \mathrm{s}$ to $300 \mathrm{~km} / \mathrm{s}$ on August $22-24$, IMF southern component fluctuations were within $\mathrm{Bz}= \pm 4 \mathrm{nT}-\mathrm{Bz}= \pm 6 \mathrm{nT}$. Due to the arrival of a inhomogeneous accelerated flux from coronal mass ejection (CME from August 20), SWV increased to $450 \mathrm{~km} / \mathrm{s}$ on August 25, IMF southern component fluctuations reached the value $\mathrm{Bz}= \pm 11 \mathrm{nT}$. At the beginning of the day on August 26, an inhomogeneous 
accelerated flux from two coronal holes arrived, SWV began to increase and reached the maximum value of $666 \mathrm{~km} / \mathrm{s}$ on August 27, IMF fluctuations grew to $\mathrm{Bz}= \pm 17 \mathrm{nT}$. Then up to the period under analysis, SWV was within $\mathrm{v}=500-550 \mathrm{~km} / \mathrm{s}$, IMF southern component fluctuations were at the level $\mathrm{Bz}= \pm 4 \mathrm{nT}$. Based on the results of neural network operation, in the second part of the day on August 24, anomalous effects of low amplitude («weaklydisturbed» class) occurred in cosmic ray dynamics. The network referred the period of August 25-27 to the «disturbed» one that corresponds to large sporadic effect occurrences. Comparison of the neural network operation results with cosmic weather data and wavelet spectrum (Fig. 3b) confirms the accuracy of network solution. Analysis of ionospheric data processing (Fig. $3 \mathrm{e}-\mathrm{g}$ ) shows a long positive anomaly occurrence (red color) before the magnetic storm. The anomaly was of low intensity with the maximum during the second part of the day on August 24 (UT). We should note that the time of ionospheric anomaly intensity maximum (Fig. 3g) coincides with the time of cosmic ray intensity increase (Fig. 3d) and IMF fluctuations increase (Fig. 3h). During the main phase of the magnetic storm, electron concentration oscillations are observed in the ionosphere. First, the concentration decreased than an «attempt» of recovery was observed (from 01.00 till 12.00 UT on August 26). From August 26 to 27, a multiscale negative (blue color) anomaly is observed in the ionosphere at the recovery phase of the magnetic storm (Fig. 3f,g). The occurred negative ionospheric storm lasted for more than one day and a half and was the most intensive at 14.00 UT on August 27. Comparison of anomalous changes in the ionosphere with IMF and SWV data during the event shows the correlation, oscillations in the ionosphere are observed during Bz component fluctuations increase and SWV growth. 


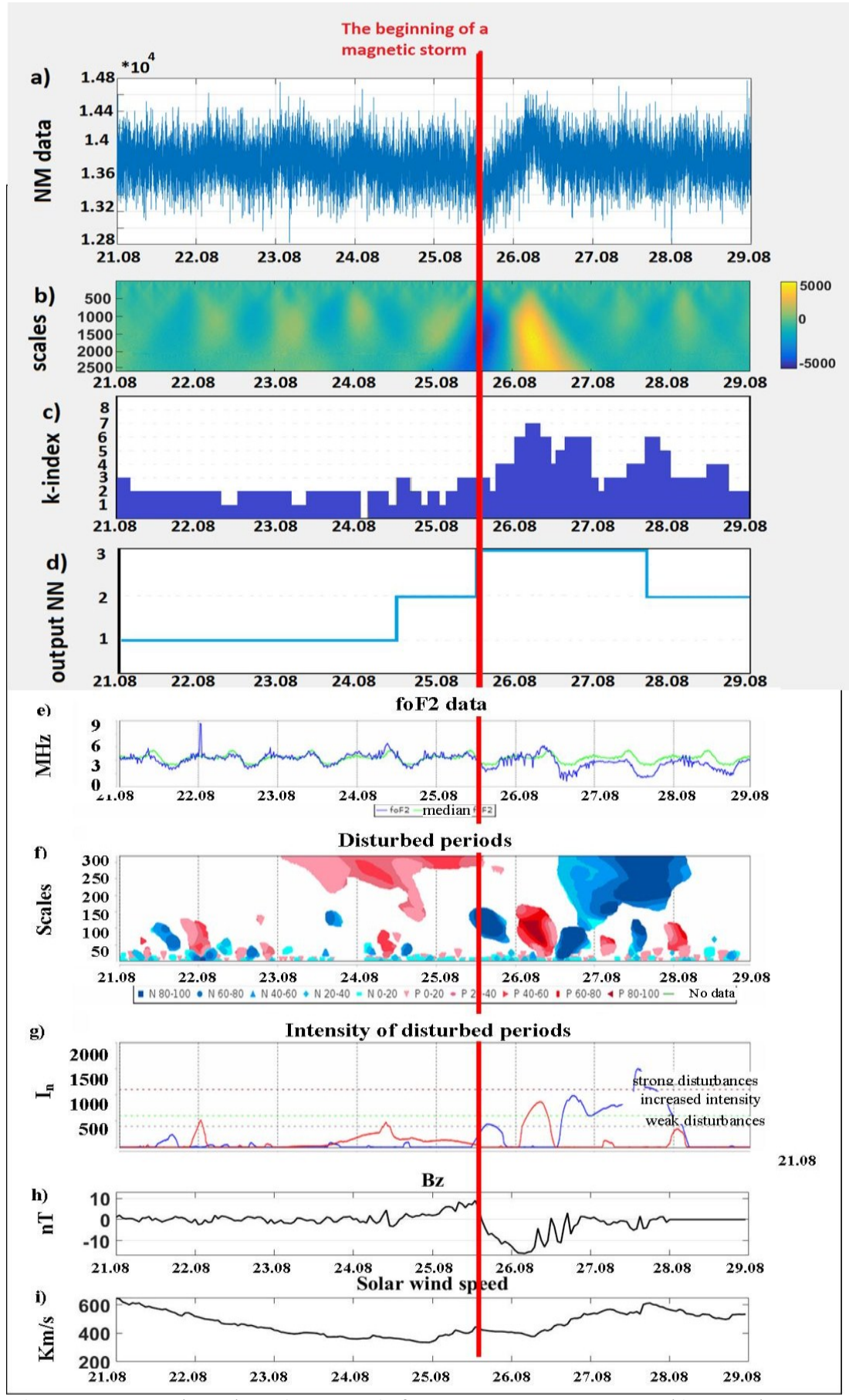

Fig. 3. a) neutron monitor data (Moscow) for August 21-28, 2018, b) wavelet spectrum of neutron monitor data, c) K-index data, d) results of neuron network LVQ_coif3_2 operation, e) foF2 data (blue) and its median values (green) (Paratunka), f) disturbe d periods in the ionosphere, g) disturbance intensity in the ionosphere, h) IMFBz, i) solar wind velocity. 
Fig. 4 illustrates the results of data processing for March 12-19, 2018. Analysis of geomagnetic activity index data (K-index [17], Fig. 4c) shows the presence of strong and long geomagnetic disturbances during the period under analysis. Owing to the arrival of an accelerated flux from a coronal hole at 11.00 UT on March 14, SWV gradually increased to $533 \mathrm{~km} / \mathrm{s}$ within a day on March 14 and 15, IMF southern component fluctuations increased to $\mathrm{Bz}= \pm 11 \mathrm{nT}$ on March 14 (Fig. 4h), SWV was within $\mathrm{v}=410-530 \mathrm{~km} / \mathrm{s}$ on March 16 and 17, IMF component was $\mathrm{Bz}= \pm 6- \pm 9 \mathrm{nT}$. From the beginning of the day on March 18 and to the end of the day on March 19, SWV increased to $624 \mathrm{~km} / \mathrm{s}$, IMF fluctuations reached $\mathrm{Bz}= \pm 10 \mathrm{nT}$ due to the arrival of an accelerated flux from the second part of a coronal hole. Based on the results of neural network operation (Fig. 4d), small-scale sporadic effects («weakly-disturbed» class) appeared in the dynamics of cosmic rays before the magnetic storm (March 12). The network referred the period of March 14-20 to the «disturbed» class that corresponds to large sporadic effects and confirms the accuracy of the solution. Ionospheric data processing results (Fig. 4e-g) show the occurrences of large-scale ionospheric anomalies during the period under analysis. A negative ionospheric anomaly of low intensity occurred during a weak magnetic storm on March 15-16 and had intensity maximum close to average statistical values during geomagnetic disturbances (Fig. 4g). The anomaly lasted for about one day and a half. At the background of anomalous decrease of electron concentration in foF2 data, oscillations (positive anomalies) of small scales are observed (Fig. $4 \mathrm{f}$, g). Similar to the event described above, changes in ionospheric parameter dynamics correlate with IMF fluctuation amplitude increases. Before the strong magnetic storm during the second half of the day on March 17, a positive anomaly occurred in the ionosphere. It reached the intensity maximum at about 02.00 UT on March 18. During the recovery phase, electron concentration in the ionosphere significantly decreased (negative anomaly on March 19). 


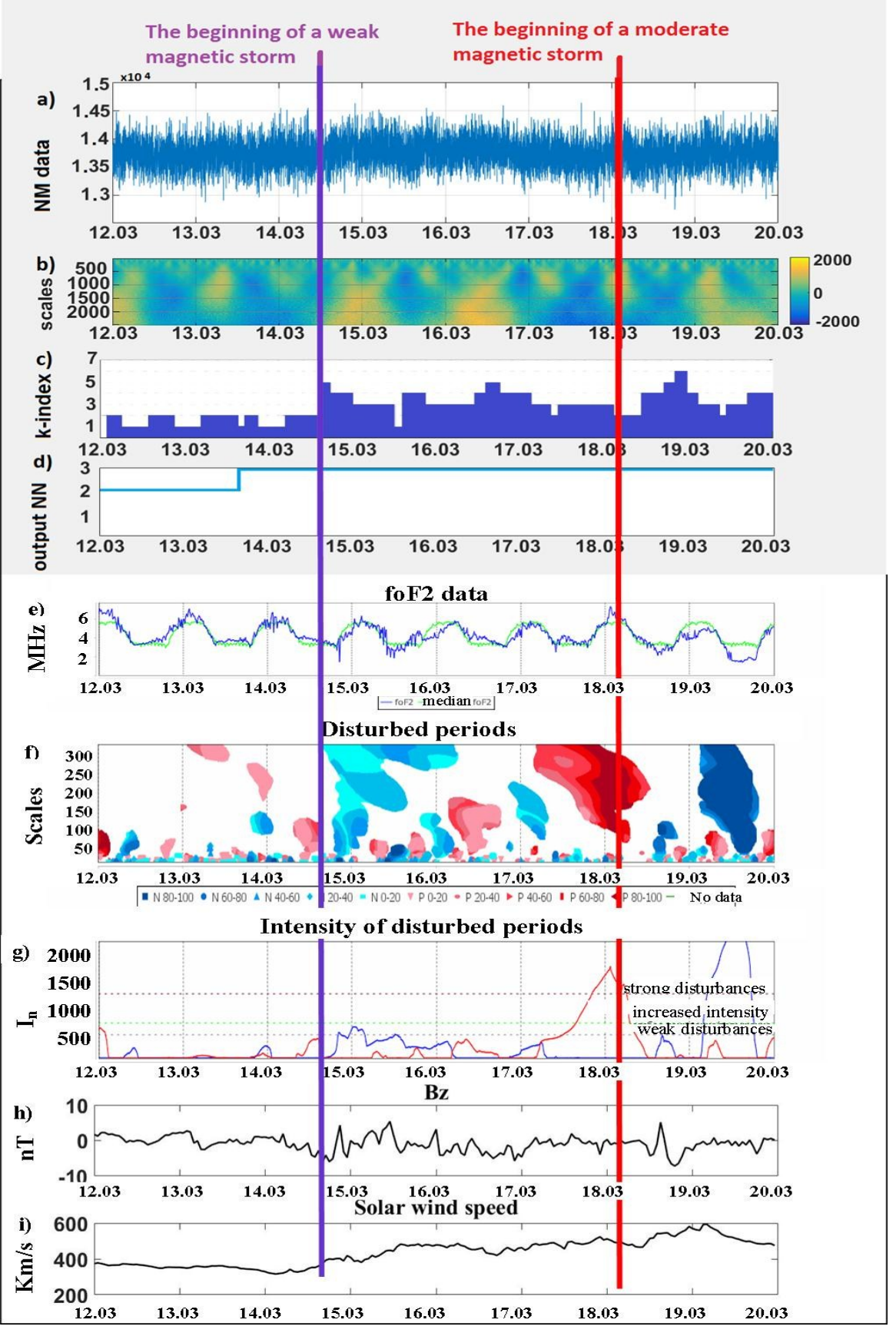

Fig. 4. a) neutron monitor data (Moscow) on March 12-19, 2018, b) wavelet spectrum of neutron monitor data, c) K-index data, d) results of neuron network LVQ_coif3_2 operation, e) foF2 data (blue) and its median values (green) (Paratunka), f) disturbed periods in the ionosphere, g) disturbance intensity in the ionosphere, h) IMFBz, i) solar wind velocity. 
Fig. 5 illustrates the results of data processing during a moderate magnetic storm on October 7, 2018. According to the space weather data [15], SWV increased from 360 to $514 \mathrm{~km} / \mathrm{s}$, IMF southern component fluctuations raised from $\mathrm{Bz}= \pm 2 \mathrm{nT}$ to $\mathrm{Bz}= \pm 5 \mathrm{nT}$ on October 3 due to the coronal hole effect. Then on October 7, SWV gradually decreased to $360 \mathrm{~km} / \mathrm{s}$, IMF southern component fluctuations fell to $\mathrm{Bz}= \pm 3 \mathrm{nT}$. At about 06.00 in the morning on October 7, an inhomogeneous accelerated flux from a coronal hole (crossed the central meridian on October 5) arrived. By the end of the day on October 7, SWV increased to $625 \mathrm{~km} / \mathrm{s}$, IMF southern component fluctuations increases to $\mathrm{Bz}= \pm 15 \mathrm{nT}$. On October 8, SWV remained within 500-620 km/sec, IMF southern component fluctuations were $\mathrm{Bz}= \pm 6$ $\mathrm{nT}$. At the end of the period, solar wind velocity grew to $632 \mathrm{~km} / \mathrm{s}$, IMF southern component fluctuations decreased to $\mathrm{Bz}= \pm 5 \mathrm{nT}$. Based on the results of neural network operation (Fig. 5d), small-scale sporadic effects were observed in cosmic ray dynamics on October 4-7 before the magnetic storm. The period from 00 on October till the noon on October 8 was referred to the «disturbed» class by the network that corresponds to large sporadic effects and confirms the accuracy of the solution. The results of ionospheric data processing (Fig. 5e-f) show the occurrence of a short positive anomaly of average intensity (at the boundary of October 5-6 (UT), Fig. 5g) before the magnetic storm. The anomaly was of short duration (within 8 hours) and had statistically high intensity for a calm period. The second positive anomaly of weak intensity occurred several hours before the magnetic storm beginning. In the vicinity of Dst amplitude maximum (Fig. 5c), electron concentration in the ionosphere decreased and a negative anomaly of average intensity occurred (anomalies are shown by blue color in Fig. $5 \mathrm{f}, \mathrm{g}$ ). During the storm recovery phase, electron concentration of the ionosphere remained decreased. The effect is likely to be determined by thermosphere warming up and lift that causes recombination rate increase and, as a sequence, ionization depletion [18]. 


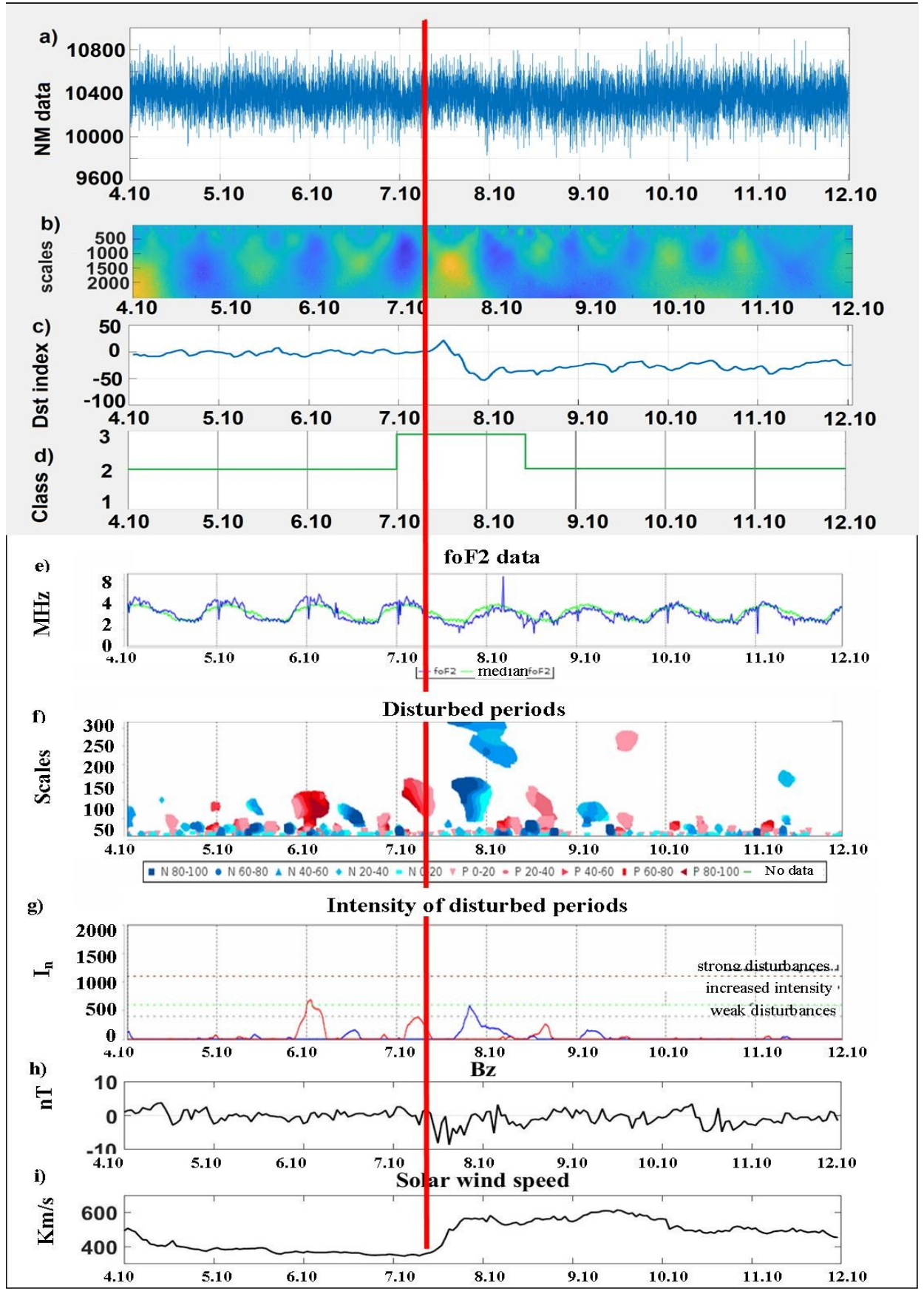

Fig. 5. a) neutron monitor data (Moscow) on October 4-11, 2018, b) wavelet spectrum of neutron monitor data, c) Dst-index data, d) results of neuron network LVQ operation, e) foF2 data (blue) and its median values (green) (Paratunka), f) disturbed periods in the ionosphere, g) disturbance intensity in the ionosphere, h) IMFBz, i) solar wind velocity.

In order to obtain quantitative estimates of the observable correlation effects in IMF parameter dynamics and in ionospheric data variations, statistical processing of events for the period of August 012015 - September 30, 2018 has been carried out and a model of dual regression between $\mathrm{Bz}$ component values and ionospheric disturbance intensity values 
has been constructed. We analyzed the periods when IMF Bz component had the values less than $-8 \mathrm{nTl}$. Taking into account the possible duration of ionospheric disturbances [19], ionospheric data were analyzed in a 3-day time window, each beginning from 00:00 UT. Thus, for the ionospheric data we processed the periods including the date of the event in IMF and the two following days. Gaps in ionospheric data were filled with median values (for 10 preceding days) taking into account the diurnal variation. When a gap was long (more than a day), the event was removed from the statistics. Gaps in IMF data were filled by the neighbor method [20]. When the total duration of gaps in IMF data was more than $30 \%$ of the period under analysis or the dap duration was $15 \%$ and more, the event was removed from the statistics.

In the course of ionospheric data processing for the period under analysis (three days for one event), maximum intensity for negative ionospehric storms was determined. As long as the duration of the analyzed periods for IMF parameters was one day and for foF2 data it was three days, the highest values for the period under analysis were used during the periods of long disturbances in IMF parameters (assumed that they contributed the most to ionospheric disturbances).

Based on the chosen criteria for the processing, 46 events were selected: 14 storms of G0 class (weakly-disturbed geomagnetic state), 10 storms of G1 class (weak geomagnetic storm), 17 storms of G2 class (moderate magnetic storm), 4 storms of G3 class (strong magnetic storm) and 1 storm of G4 class (very strong magnetic storm). These events corresponded to negative ionospheric storms of different intensity. Events with positive ionospheric storms were not included into the analysis. Correlation estimated coefficient is

$$
r=\frac{\sum_{i}\left(x_{i}-\bar{x}\right)\left(z_{i}-\bar{z}\right)}{\sqrt{\sum_{i}\left(x_{i}-\bar{x}\right)^{2} \sum_{i}\left(z_{i}-\bar{z}\right)^{2}}}=0.6,
$$

where $\bar{x}=\frac{1}{n} \sum_{i} x_{i}, \bar{z}=\frac{1}{n} \sum_{i} z_{i}$

The obtained model of dual regression has the form

$$
Z=-14.86 x_{B z}-39.24
$$

In order to estimate the model quality and to check the statistical significance of estimated parameters, we calculated the following characteristics:

- Critical Student's distribution with $k=(n-2)$ degree of freedom (where the number of events is $n=46): t_{c r}=t(0.95,44)=2.01$.

- Sample t-statistics, estimated for regression coefficient Bz $t_{B z}=\frac{|r|}{\sqrt{1-r^{2}}} \sqrt{n-2}=-4.98$.

The obtained characteristics confirmed the statistical significance of the estimated parameters and model validity that indicated the statistically significant effect of IMF parameters on ionospheric data parameters (confidence coefficient is $95 \%$ ).

\section{Conclusions}

The method suggested in the paper to analyze neutron monitor data has shown its efficiency when applied in the tasks of investigation of cosmic ray dynamics and detection of sporadic effects. On the example of considered events for 2018, the possibility of application of the method in the problems of real-time analysis of neutron monitor data and detection of different-scale sporadic effects, including those of low amplitude, has been confirmed. 
The method for ionospheric parameter analysis allowed us to investigate ionospheric process dynamics during disturbed periods in detail. Ionospheric disturbances were detected in the regions under analysis. These disturbances preceded and accompanied magnetic storm periods. The analysis confirmed the possibility of occurrence of pre-increase effect in the ionosphere that agrees with multiple observations described in the papers $[9,10,11]$. Comparison with cosmic ray data and IMF parameters gives us the ground to assume solar nature of the detected anomalous effects in the ionosphere.

During the magnetic storm main phases, oscillations, correlating with IMF variations, were observed in the ionosphere. Statistical significance of IMF parameter effect on ionospheric data dynamics and ionospheric anomaly occurrences has been quantitatively confirmed (confidence coefficient is 95\%). At the magnetic storm recovery phase, ionospheric process dynamics changed and anomalous decrease of electron concentration was observed. It is likely to be determined by thermosphere warming up and lift causing recombination rate increase and, as a sequence, ionization depletion [18].

In the future the authors plan to continue the investigation in this direction applying a wider spectrum of data recording sites and extending the statistical material.

\section{References}

1. L.I. Dorman,Variations of cosmic rays and study of the cosmos (Foreign Technology Division, Ohio, 1966).

2. http://cr0.izmiran.ru/common/links.htm(01.09.2019).

3. V. Vipindas, S. Gopinath, T.E. Girish, Astrophys. Space Sci., 361 (2016).

4. A. Mishev, I. Usoskin, XXV European cosmic ray symposium, eConf C16-09-04.3 (2016).

5. A.V. Belov, J.W. Bieber, E.A. Eroshenko, P. Evenson, R. Pyle, V.G. Yanke, Space Res., 31(4), 919-924 (2003).

6. S. Haykin, Neural networks and learning machines (New Jersey, Prentice Hall, 2009).

7. V.A. Golovko, Neural networks: training, organization and application (Moscow, IPRZhR, 2001).

8. T. Kohonen, Self-organizing maps (Heidelberg, Berlin, 2001).

9. A.D. Danilov, Advances in Space Research, 52, 343-366 (2013).

10. O.V. Mandrikova, N.V. Fetisova, Y.A. Polozov, I.S. Solovev, M.S. Kupriyanov, Earth, Planets and Space, 67 (2015).

11. O. Mandrikova, Yu. Polozov, N. Fetisova, T. Zalyaev, Journal of Atmospheric and Solar-Terrestrial Physics, 181, 116-126 (2018).

12. O.V. Mandrikova, N.V. Fetisova (Glushkova), R.T. Al-Kasasbeh, D.M. Klionskiy, V.V. Geppener, M.Y. Ilyash, Annals of Geophysics, 58 (2015).

13. S. Mallat, A wavelet tour of signal processing (Academic Press, London, 1999).

14. N.P. Baldin, Machine learning and data analysis, 1(1), 61-76 (2011).

15. Space weather forecast according to the Institute of Applied Geophysics, http://ipg.geospace.ru (01.09.2019).

16. O.V. Mandrikova, V.V. Geppener, B.S. Mandrikova, Journal of Physics: Conference Series (to be published).

17. http://ipg.geospace.ru/3h-k-index/ (01.09.2019).

18. M. Nakamura, T. Maruyama, Y. Shidama, Earth, Planets Sp., 59 (2007).

19. S. Li, R. Galas, D. Ewert, J. Peng, Acta Geophys., 64(1), 253-269 (2016).

20. R.J.A. Little, D.B. Rubin, Statistical analysis with missing data (Wiley, N.-Y., 2002). 